\title{
Research on the Evaluation of Customer requirement Importance of Smart Products Based on Online Comments and Improved Quantitative Kano Model
}

\author{
Hang Liu*, Zan Ren, Yingjie Li \\ School of Management Engineering, Zhengzhou University of Aeronautics, Zhengzhou,Henan, China \\ *Corresponding Author.
}

\begin{abstract}
With the development and popularization of smart products, the technological differences of products are decreasing, and the phenomenon of product homogeneity is becoming more and more obvious. It is necessary for the smart product manufacturing firms have the capability to analyze customer requirement deeply and adapt to the dynamically changing market quickly. Therefore, the traditional technology-oriented product development model is no longer suitable for manufacturers to obtain a competitive advantage. Based on this, this paper proposed a method to evaluate the importance of customer demands based on online comments and quantitative Kano model. First, the Python crawler tool is used to obtain online customer reviews of relevant products and the word segmentation processing is performed to obtain the product features and frequency that customers are mainly concerned about, and then the initial importance of demand can be calculated. Furthermore, use the quantitative Kano model to determine the customer satisfaction and revise the initial importance of the requirements to obtain a more reasonable ranking of the importance of user needs. Finally, a case study is carried out with the smart bracelet as an example to verify the effectiveness and feasibility of the model proposed in this paper.
\end{abstract}

Keywords: online comments, improved quantitative Kano model, requirement importance

\section{Introduction}

Traditional product development is technology-oriented [1]. With the development and popularization of smart products, the technological differences of products are decreasing, and the phenomenon of homogeneity is becoming more and more obvious. In-depth understanding and analysis of the individual needs of users, and quickly adapting to the dynamic market have become a key path for modern manufacturing companies, especially smart product manufacturers, to gain competitive advantage. Therefore, accurate customer demand analysis has become more important in the product development process, and has become a core factor to enhance the company's core competitiveness [2,3]. Customer demand analysis, usually conducted at the early stage of product development, is the basis of the future detailed design and a crucial step to determine whether the product can have an excellent performance in the future market or not. Based on the demand-oriented design concept, this paper proposes a method for determining the importance of customer demand that uses the quantitative Kano model to modify the initial importance of demand.

Domestic and foreign scholars have conducted certain researches on the field of determining the importance of customer requirements [4-7], such as network analysis, Kano model, rough set and information entropy processing. Zhu and Yang [8] proposed the DEMATEL method and entropy to deal with the autocorrelation and market competitiveness of customer needs, and combined the results of these two aspects to get the final importance ranking. Liu and Wang et al [9] used the combination method of AHP and fuzzy comprehensive evaluation to construct analysis models of different demand elements, and quantifiedcustomer requirements through ranking scale.Geng and Qiu [10] combined hesitating fuzzy sets and decision-making experiments with evaluation experiments to analyze customer requirements, and proposed an improved A-Kano model to modify the customer requirements. Ji [11] analyzed the relationship between customer needs and satisfaction, quantitatively integrated the Kano model with QFD, and formulated a mixed nonlinear integer programming model.

ISSN: 0010-8189

(C) CONVERTER 2021 
Summarizing the existing literature review, it can be found that in the customer requirements acquisition and identification stage, most of the literatures [12-14] use Fuzzy Comprehensive Evaluation, Analytic Hierarchy Process and other expert scoring methods, which are highly subjective and lack the Voice of Customer. Therefore, based on the customer-centered concept, this article obtains the real needs of users through direct acquisition and analysis of online customer comments, ensuring the accuracy and reliability of the data.

The framework is shown in Figure 1 below. After collecting and identifying customer requirements, the initial importance of customer requirements can be calculated.Then, quantifying the demands classification of Kano model, and customer satisfaction can be calculated by using the processed model. Finally, comparing and revising it with the initial importance, the final importance ranking can then be obtained.

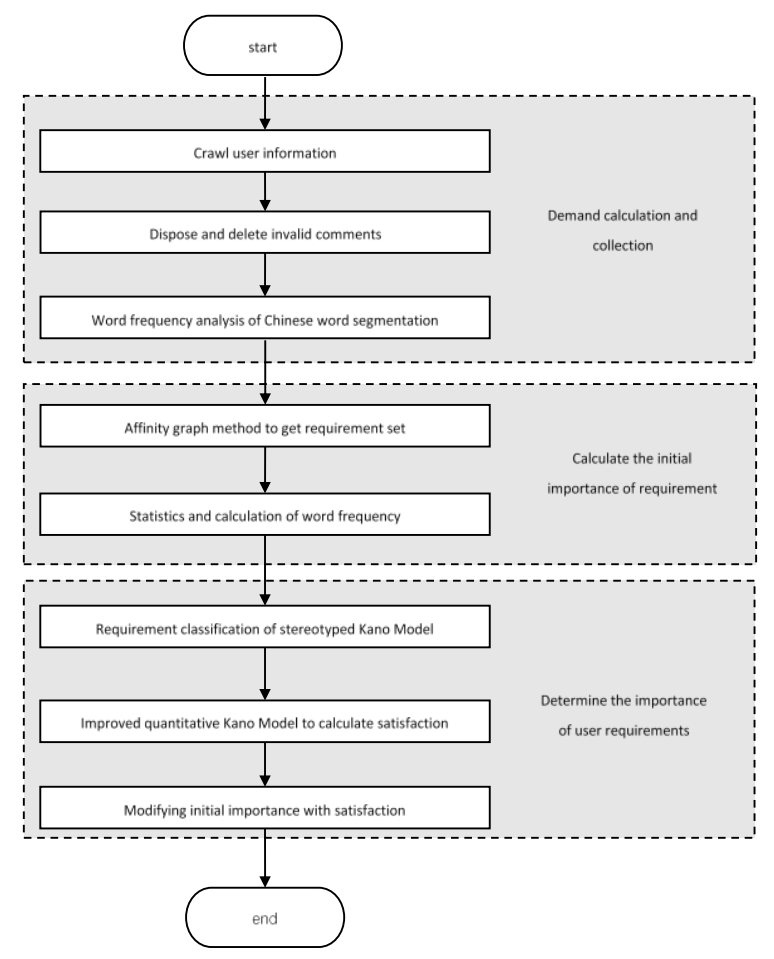

Figure1. The framework

\section{CustomerRequirements Collectionand Initial Importance Identification}

Traditional customer requirements acquisition methods mainly include questionnaire surveys and user interviews, which have obvious disadvantages such as high research costs and difficulty in obtaining sufficient samples. In order to ensure the objectivity and diversity of the data, this article uses Python crawler tools to crawl customer comments data of related smart products from the e-commerce platform. Through analyzing and processing of the comment data, the characteristics of smart products that customersconcern can be obtained directly.These characteristics will be transformed into customer requirements in next step.

When collecting customer requirements, the first step is to de-duplicate the data obtained with Python, and use the word frequency analysis method to obtain the needs concerned by customers [14]. Some words such as "not bad", "ok"and "good" can often be seen in product reviews. These words can be used to describe various types of products, rather than a specific characteristic of a certain product. These words make it difficult to identify specific user needs accurately. Therefore, the word frequency analysis needs to be filtered. The specific words that can reflect customer demands are selected afterfiltering out some common fuzzy expression, and then a word map can be drawn to calculate the frequency.

ISSN: 0010-8189 
The high-frequency vocabulary selected based on the results of word frequency analysis is fuzzy, which cannot be used in product design directly. For example, the high-frequencydescription of the size requirement is "long", "very short", "too wide", etc. These words need to be further abstracted and turned into the length characteristic of "size", which can indicate whether it is suitable for carrying.

This paper uses KJ method to summarize and organize high-frequency words. The main steps includes: (1) determine the theme; (2) collect the corpus; (3) cardize the corpus; (4) group the cards; (5) collect the cards; (6) make the cards; (7) arrange the cards; (8) ) Complete the KJ map. The specific operation will be carried out in the case study.

Through the observation of real customer reviews, it can be found that the more frequently a certain demand characteristic appears, the demand is more concerned by customers.In other words, the demand frequency can intuitively express the importance of the relative demand characteristic. Therefore, this paper calculates the initial importance of customer requirements according to the frequency of each demand.

The customer demand set obtained through the $\mathrm{KJ}$ method is recorded as $\mathrm{C}=\left\{\mathrm{c}_{1}, \mathrm{c}_{2}, \mathrm{c}_{3}, \ldots, \mathrm{c}_{\mathrm{n}}\right\}$, the demand frequency set is recorded as $\mathrm{CV}=\left\{\mathrm{cv}_{1}, \mathrm{cv}_{2}, \mathrm{cv}_{3}, \ldots, \mathrm{cv}_{\mathrm{n}}\right\}$. Set the initial importance of the demand as $\mathrm{cp}^{\alpha}$, then the frequency of the requirement characteristic $\mathrm{C}_{\mathrm{i}}$ can be calculated as:

$$
c p_{i}^{\alpha}=\frac{c v_{i}}{\sum_{i=1}^{n} c v_{i}}(3)
$$

After acquiring the frequency of each demand characteristic, theinitial customer requirement importance set can be obtained as:

$$
C P^{\alpha}=\left\{c p_{1}^{\alpha}, c p_{2}^{\alpha}, c p_{3}^{\alpha}, \ldots, c p_{n}^{\alpha}\right\}(4)
$$

\section{Modification of the Initial Importance of Customer Requirements}

\subsection{Improved Quantitative Kano Model}

The Kano model is a well-known model in the research field of customer requirements analysis and productdesign. For a certain customer demand or the corresponding quality attribute $\mathrm{C}_{\mathrm{i}}$, use $\mathrm{A}, \mathrm{O}, \mathrm{M}, \mathrm{I}$ and $\mathrm{R}$ to represent charmrequirements, expectationrequirements, basic requirements, indifference requirements and opposite requirements respectively, and Q represents invalid answers.

Collect the survey results of all customers, summarize for a certain fixed requirements, record its frequency, use $V_{A}$ 、 $V_{O} 、 V_{M} 、 V_{I} 、 V_{R}$ to represent the frequency of charm requirements, expectation requirements, basicrequirements, indifferencerequirements and opposite requirements respectively. $V_{Q}$ represents the frequency of invalid answers, let the total frequency be $\mathrm{V}$ :

$$
V=V_{A}+V_{O}+V_{M}+V_{R}+V_{I}+V_{Q}(1)
$$

Kano classification is the requirements classification corresponding to the maximum frequency, denoted as MC:

$$
M C=\max \left(V_{A}+V_{Q}+V_{O}+V_{M}+V_{R}+V_{I}\right)
$$

ISSN: 0010-8189 
Table 1.Requirement Classification Table

\begin{tabular}{ccccccccc}
\hline Requirement & $\mathrm{A}$ & $\mathrm{M}$ & $\mathrm{O}$ & $\mathrm{R}$ & $\mathrm{Q}$ & $\mathrm{I}$ & Total & Kano Classfication \\
\hline $\boldsymbol{C}_{\mathbf{1}}$ & $V_{A}^{1}$ & $V_{M}^{1}$ & $V_{O}^{1}$ & $V_{R}^{1}$ & $V_{Q}^{1}$ & $V_{I}^{1}$ & $V^{1}$ & $M C^{1}$ \\
$\ldots \ldots$ & & & & & & & & \\
$\boldsymbol{C}_{\boldsymbol{i}}$ & $V_{A}^{1}$ & $V_{M}^{1}$ & $V_{O}^{1}$ & $V_{R}^{1}$ & $V_{Q}^{1}$ & $V_{I}^{1}$ & $V^{i}$ & $M C^{i}$ \\
\hline
\end{tabular}

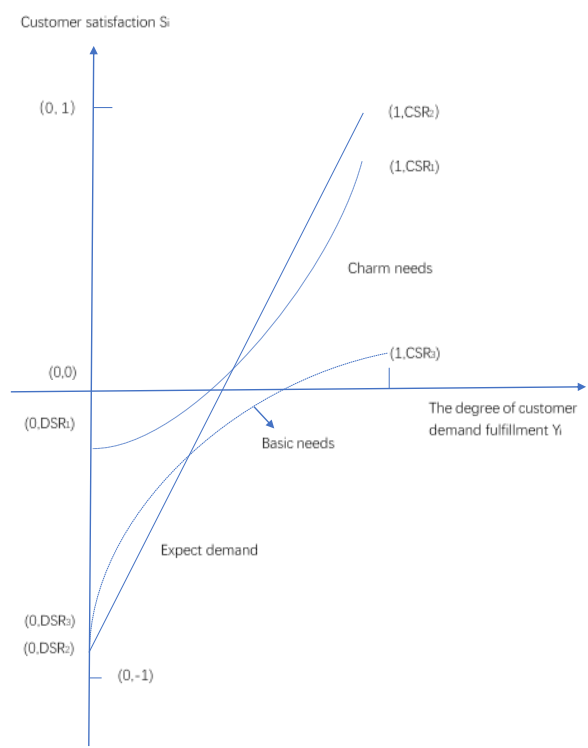

Figure 2. Improved quantitative Kano model

The current quantitative Kano model [6] is shown in Figure 2. The expressions of the three curves are shown in formula (5):

$$
S_{\mathrm{i}}= \begin{cases}\frac{C S R_{i}-D S R_{i}}{e-1} e^{y_{i}}-\frac{C S R_{i}-e D S R_{i}}{e-1}, & \text { Charm needs } \\ \left(C S R_{i}-D S R_{i}\right) y_{i}+D S R_{i}, & \text { Expect demand } \\ -\frac{e\left(C S R_{i}-D S R_{i}\right)}{e-1} e^{-y_{i}}+\frac{e C S R_{i}-D S R_{i}}{e-1}, & \text { Basic needs }\end{cases}
$$

The quantitative Kano model in Figure 2 has two important parameters: the customer satisfaction index CSR, and the customer dissatisfaction index DSR. This parameter can be calculated by the formula (6) and (7).

$$
\begin{aligned}
& C S R_{i}=\frac{V_{A}^{i}+V_{O}^{i}}{V_{A}^{i}+V_{O}^{i}+V_{M}^{i}+V_{I}^{i}}(6) \\
& D S R_{i}=\frac{-\left(V_{O}^{i}+V_{M}^{i}\right)}{V_{A}^{i}+V_{O}^{i}+V_{M}^{i}+V_{I}^{i}}(7)
\end{aligned}
$$

ISSN: 0010-8189 
The quantitative Kano model is the quantitative expression of the traditional Kano model, which should not change the demand classification and characteristics. However, the current quantitative Kano model has the following shortcomings:

(1) The traditional Kano model describes the demand for charm requirement as: the presence or absence of this demand will not reduce customer satisfaction. Combining the quantitative model curve and equation (5), the charm demand curve crosses the point $\left(0, \mathrm{DSR}_{\mathrm{i}}\right)$, and according to equation (6), DSR $<0$. The point $\left(0, \mathrm{DSR}_{\mathrm{i}}\right)$ represents that the user's dissatisfaction is negative when the objective performance of the product is zero, which contradicts the concept of charmrequirement.

(2)The traditional Kano model describes the basic requirement as: the requirement should be realized, but the degree of customer satisfaction will not change with the realization of the requirement. However, according toFigure 2 and equation (5), the curve of basic requirement passes the point $\left(1, \operatorname{CSR}_{\mathrm{i}}\right)$, which means that when the

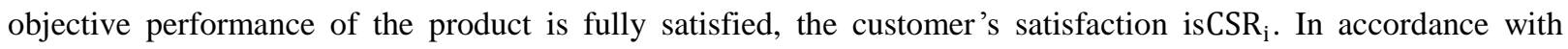
equation (6), $\operatorname{CSR}_{\mathrm{i}}$ is greater than 0 , which does not match the concept of basic requirement.

Based on the above analysis, the current quantitative Kano model should be revised to make it consistent with the demand planning. Let $\mathrm{A}, \mathrm{O}$, and $\mathrm{M}$ respectively represent the set of charm requirement, expectation requirement and basic requirement, the mathematical expression of customer satisfaction is as follows:

$$
s_{i}=\left\{\begin{array}{c}
\ln \left(\left(1-e^{D S R_{i}}\right) y_{i}+e^{D S R_{i}}\right), \text { If } i \in M \\
\left(C S R_{i}-D S R_{i}\right) y_{i}+D S R_{i}, \text { If } i \in O \\
\frac{C S R_{i}}{e-1} e^{y_{i}}-\frac{C S R_{i}}{e-1}, \text { If } i \in A
\end{array}\right.
$$

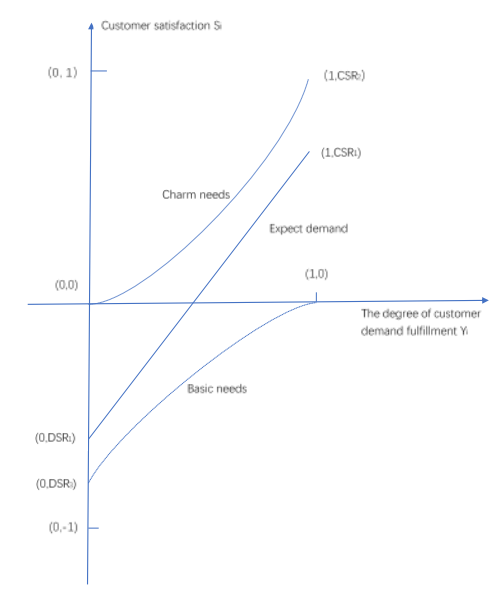

Figure 3. The quantitative Kano model is improved

From the above figure 3, it is clear that the customer satisfaction will not show negative when the charm demand cannot be met, the user satisfaction index will not be positive when all the basic requirements are fulfilled.

\subsection{Modification ofinitial importance of requirements}

Through the improved Kano model, all requirements are represented by a mathematical model to quantify the impact of different types of demands on customer satisfaction. The ranking of initial importance of users can then be revised according to the degree of customer satisfaction.

ISSN: 0010-8189 
The initial importance of requirements should follow the principle that customer satisfaction is inversely proportional to the importance of product improvement demands. Higher the customer satisfaction means less improvement is needed. Therefore, the higher the customer satisfaction, the lower the importance of product improvement demands.

Set the customer satisfaction of a certain product requirement is $C S=\left\{s_{1}, s_{2}, \ldots, s_{n}\right\}$. The modified importance degree set of demand is denoted as $C P^{\beta}=\left\{c p_{1}^{\beta}, c p_{2}^{\beta}, \ldots, c p_{n}^{\beta}\right\}$. According to Equation (3), the initial importance degree and satisfaction degree of customer demand are both valued as $(0,1)$. In accordance with the above principle and the basic properties of power function, $C P^{\beta}$ can be calculated by Equation (7).

$$
c p_{i}^{\beta}=\frac{\left(c p_{i}^{\alpha}\right)^{s_{i}}}{\sum_{i=1}^{n}\left(c p_{i}^{\alpha}\right)^{s_{i}}}(9)
$$

Where $s_{i}$ is the user satisfaction, according to $\mathrm{y}=a^{x}$, when $\mathrm{a} \in(0,1), \quad \mathrm{y}$ and $\mathrm{x}$ are inversely proportional.

\section{Case Study}

This paper takes the smart bracelet as an example. In order to make the products comparable, the following constraint conditions are set when selecting the relevant products: similar price, well-known brands and multi-functional. Based on the above conditions, three smart bracelets were selected: Huawei Band 4, Xiaomi Band 6, and Joy Band 5s, which are respectively denoted as $Z_{1}, Z_{2}, Z_{3}$.

\section{1 collection and identification of customer requirements}

A total of 29,937 customer comment records were crawled by using Pycharm. After extracting the evaluation information, the text should be processed to eliminate duplicate and invalid evaluations to ensure the validity of the information. Word segmentation is performed on the comment information, word frequency is counted, and customer requirements are extracted. This step is processed with a word cloud generation tool.

The top 100 vocabularies is extracted by the word cloud generation tool and a word cloud diagram can then be obtained as shown in Figure 4. According to the principle of word cloud image generation, the more frequently a word appears, the more prominent the display specification. The main concerns of customers can be seen intuitively.

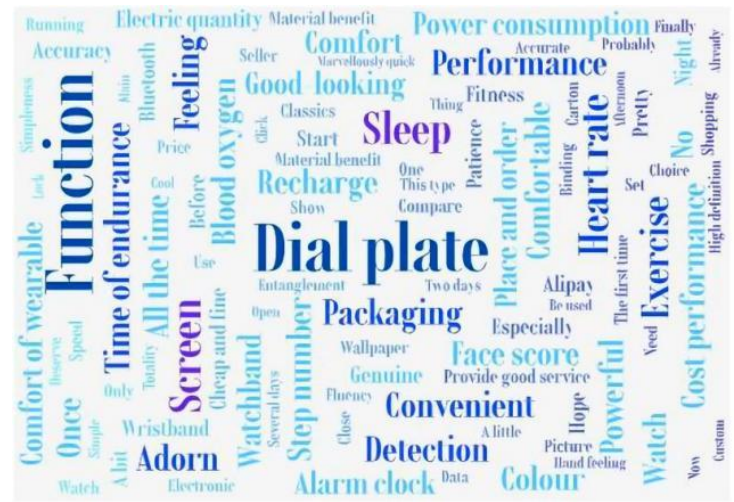

Figure 4. User evaluation word cloud chart

4.2 Customer requirement identification

When users comment on a certain product, they rarely put forward professional wordsand cannot be directly used

ISSN: 0010-8189

(C) CONVERTER 2021 
as the user's demand characteristics [15]. Therefore, the KJ method is conducted to collect and organize the frequent vocabulary. For example, words such as "heart rate", "exercise", "steps", "blood oxygen", "powerful function", "alarm clock", "fitness", "running"can all be attributed to the functional characteristics KJ card, as shown in Figure 5.

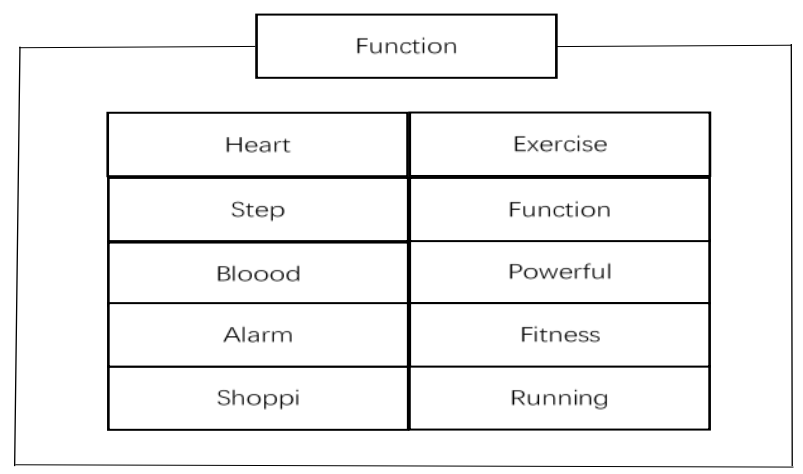

Figure 5. User requirements for "functional" affinity cards

By using the KJ graph to sort out the high-frequency vocabulary, six customer requirements: function $\left(C_{1}\right)$, appearance $\left(C_{2}\right)$, comfort of wearable $\left(C_{3}\right)$, cost performance $\left(C_{4}\right)$, time of endurance $\left(C_{5}\right)$, and packaging $\left(C_{6}\right)$.The KJ diagram of customer requirements can be acquired when putting all the KJ cards together. The result is shown in Figure6 below.

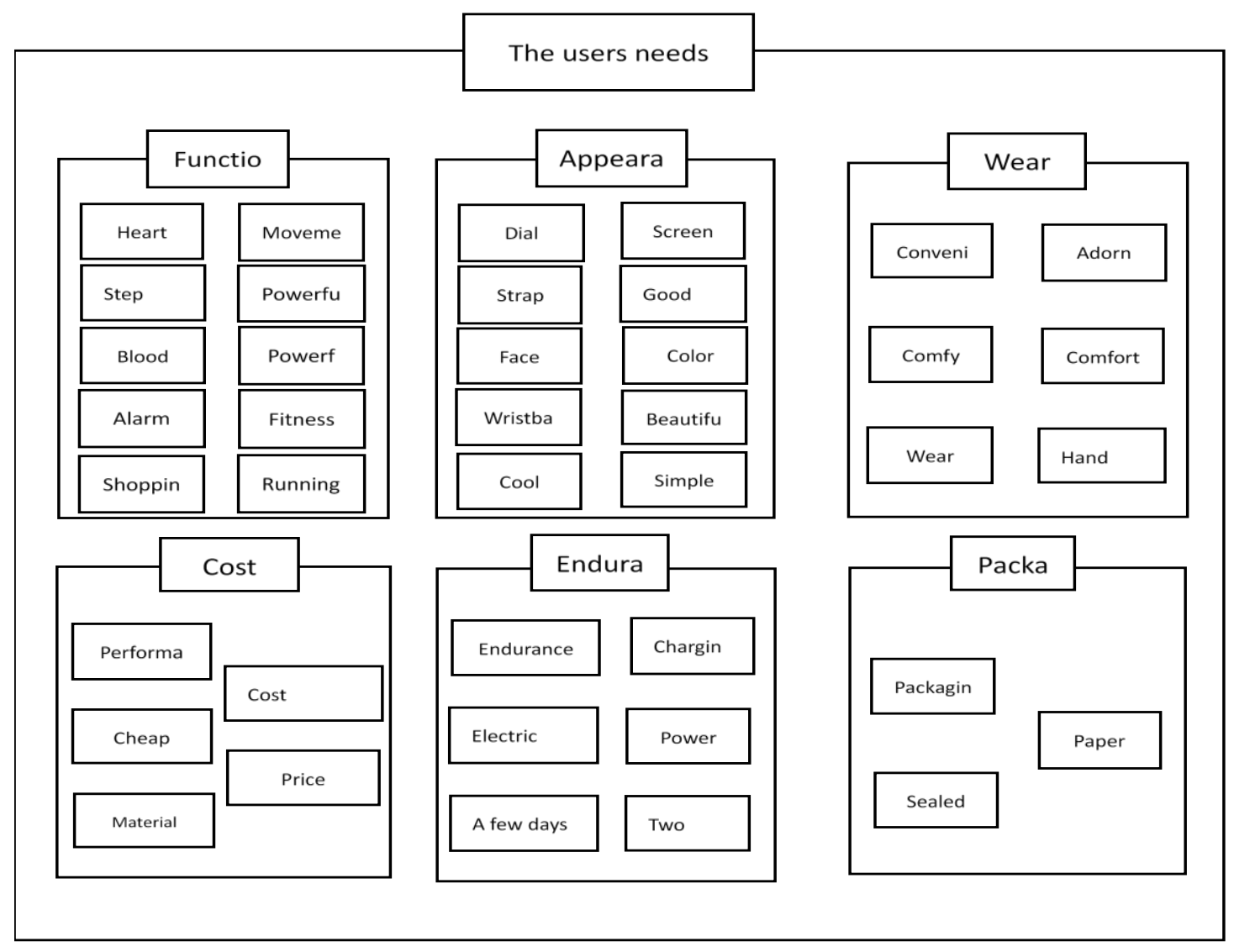

Figure 6. User Requirement Affinity Diagram

ISSN: 0010-8189 


\subsection{Calculation of initial requirement importance}

Through the process of demand identification, the customer requirement set $C=\left\{C_{1}, C_{2}, C_{3}, C_{4}, C_{5}, C_{6}\right\}$ is obtained, and the frequency set of customer demand is recorded as $C V=\left\{c v_{1}, c v_{2}, c v_{3}, c v_{4}, c v_{5}, c v_{6}\right\}$, the frequency of each demand needs to be calculated.The customer demand frequency set is $\mathrm{CV}=\{14828,11907,6408,4097,5707,2905\}$, and the total frequency is 45852 times. According to formula (3), the initial value of the importance of each demand characteristic can be calculated.Taking $C_{1}$ as an example:

$$
c p_{1}^{\alpha}=\frac{c f_{1}}{\sum_{i=1}^{6} c f_{i}}=\frac{14828}{45852}=0.3234
$$

Similarly, the initial importance of each requirement can be obtained, and the initial importance set is:

$$
C P^{\alpha}=\{0.3234,0.2597,0.1398,0.0894,0.1245,0.0634\}
$$

4.4 Modification of importance of customer requirement

A Kano questionnaire was designed for the six identified customerneeds. Thesmart bracelet users were surveyed to obtain the attributes of each need.

\begin{tabular}{|c|c|c|c|c|c|c|}
\hline Requirement & A & $\mathrm{O}$ & M & I & Total & Kanocategory \\
\hline$C_{1}$ & 14 & 58 & 29 & 8 & 109 & $\mathrm{O}$ \\
\hline$C_{2}$ & 57 & 28 & 19 & 5 & 109 & $\mathrm{~A}$ \\
\hline$C_{3}$ & 18 & 26 & 58 & 7 & 109 & M \\
\hline$C_{4}$ & 16 & 62 & 26 & 5 & 109 & $\mathrm{O}$ \\
\hline$C_{5}$ & 28 & 60 & 14 & 7 & 109 & $\mathrm{O}$ \\
\hline$C_{6}$ & 58 & 30 & 11 & 10 & 109 & A \\
\hline
\end{tabular}

Table 2. Kano classification of customer requirement

The questionnaire is distributed to customers who are using or have used smart bracelets. A total of 650 paper questionnaires were issued and 620 questionnaires were returned. After removing invalid questionnaires and questionnaires, there were 609 valid questionnaires, with an effective rate of $93 \%$.

Through the statistical analysis of the results of the questionnaire, the classification of user needs is obtained and summarized in table 2. According to these data, function,cost performance, time of enduranceare expected requirements $\mathrm{O}$, comfort of wearable is basic requirementsA, appearance requirements, and packaging requirements are glamour requirements $M$.

\subsection{Calculation of customer satisfaction}

The customer satisfaction index $C S R_{i}$ and customer dissatisfaction index $D S R_{i}$ can be obtained by formulas (6) and (7).

$$
\begin{gathered}
C S R_{i}=\frac{V_{A}^{i}+V_{O}^{i}}{V_{A}^{i}+V_{O}^{i}+V_{M}^{i}+V_{I}^{i}}=\frac{14+58}{109}=0.66 \\
D S R_{i}=\frac{-\left(V_{O}^{i}+V_{M}^{i}\right)}{V_{A}^{i}+V_{O}^{i}+V_{M}^{i}+V_{I}^{i}}=\frac{-(58+29)}{109}=-0.80
\end{gathered}
$$

ISSN: 0010-8189 
Because the requirement $C_{1}$ is the expected demand $\mathrm{O}$, according to formula (5), the values of $C S I_{i}$ and $D S I_{i}$ are substituted, so there is a satisfaction expression for $C_{1}$ :

$$
s_{1}=\left(C S R_{i}-D S R_{i}\right) y_{1}+D S R_{i}=1.46 y_{1}-0.8
$$

\section{Table 3. Customer satisfaction of $S_{1}$}

\begin{tabular}{|c|c|c|c|c|c|c|}
\hline Requirement & $\begin{array}{c}\text { Kano } \\
\text { Category }\end{array}$ & $C S R$ & DSR & Expression & $y_{i}$ & $\boldsymbol{s}_{i}$ \\
\hline$C_{1}$ & $\mathrm{O}$ & 0.66 & -0.80 & $1.46 y_{1}-0.8$ & 0.74 & 0.28 \\
\hline$C_{2}$ & $\mathrm{~A}$ & 0.77 & -0.43 & $0.44 e^{y_{2}}-0.44$ & 0.67 & 0.42 \\
\hline$C_{3}$ & M & 0.41 & -0.77 & $\ln \left(0.54 y_{3}-0.46\right)$ & 0.65 & -0.2 \\
\hline$C_{4}$ & $\mathrm{O}$ & 0.71 & -0.81 & $1.52 y_{4}-0.81$ & 0.72 & 0.28 \\
\hline$C_{5}$ & $\mathrm{O}$ & 0.81 & -0.69 & $1.50 y_{5}-0.69$ & 0.69 & 0.35 \\
\hline$C_{6}$ & $\mathrm{~A}$ & 0.81 & -0.38 & $0.48 e^{y_{2}}-0.48$ & 0.71 & 0.50 \\
\hline
\end{tabular}

Where $y_{1}$ represents the objective performance of the product. In Kano classification, $C_{1}$ is considered to be an expected demand, but there are still 29 results that classified $C_{1}$ as a basic demand, which cannot be ignored. According to the nature of Kano model, even if the meet of basic demand has little impact on customer satisfaction. Therefore, when the degree of demand realization is 1 , customer satisfaction may not be equal to 1 , and the same as other demand classifications. after statistical calculation, the quality level of $C_{1}$ is 0.74 . Substituting into equation (10), it can be obtained that customer satisfaction is 0.28 . In the same way, the other results can be obtained, as shown in Table 3.

Taking $C_{1}$ as an exampleto modify the initial importance set by using formula (9) and the result in Table 3.The result is:

$$
c p_{1}^{\beta}=\frac{\left(c p_{1}^{\alpha}\right)^{s_{1}}}{\sum_{i=1}^{6}\left(c p_{i}^{\alpha}\right)^{s_{i}}}=\frac{0.3234^{0.28}}{4.0215}=0.1813
$$

Using the data in Table 3 to modify the importance of other requirements, the new importance set is obtained as follows:

$$
C P^{\beta}=\{0.1813,0.1411,0.3686,0.1265,0.1199,0.0626\}
$$

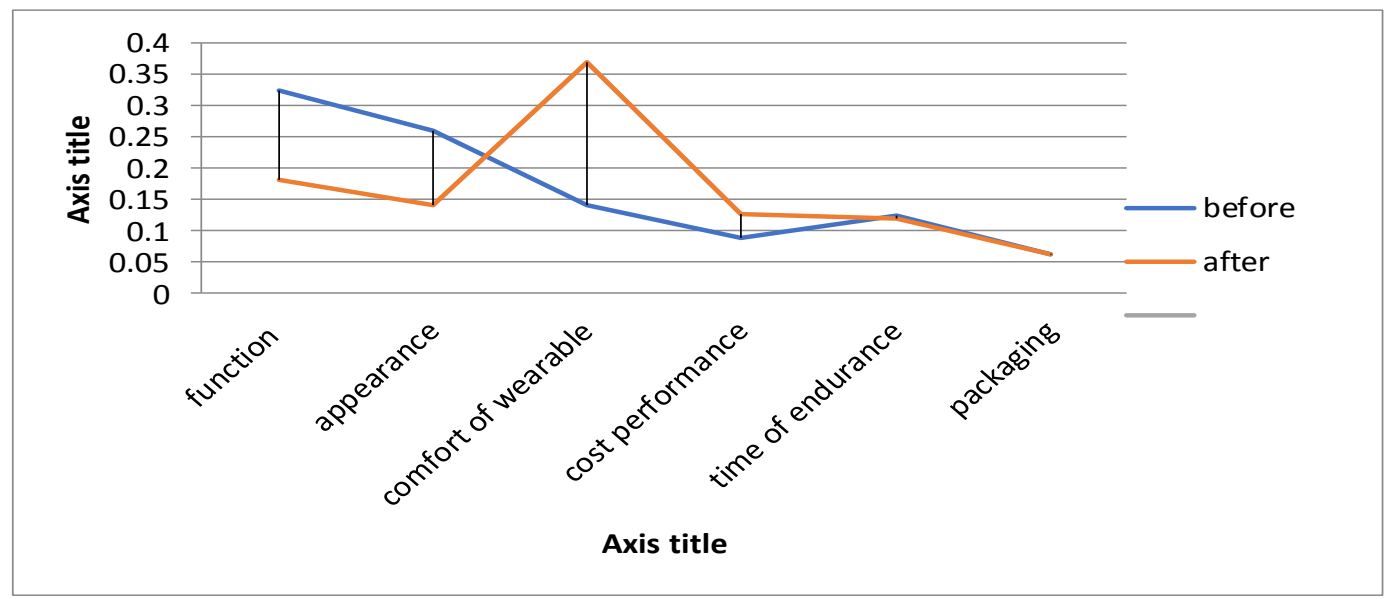

Figure 7. Comparison before and after the revision of user demand importance

ISSN: 0010-8189 
The figure 7 illustrates that after using the modified quantitative Kano model, the importance of the basic needs "comfortofwearable" has changed from the third to the first. Therefore, if the manufacturers just rely on the initial importance of requirements, the importanceof the basic requirement of"comfortofwearable" will be ignored. The result shows the modification is necessary.

\section{Conclusion}

With the advances of information technologies, many computer-based systems have been developed and employed to improve product design quality. In recent years, manufacturers are moving towards strategies to take the customers part in the design process. To address the problem mention above, this paper studies a method for evaluating the importance of customer requirements for smart products. The Python crawler tools and word frequency analysis methods are applied to obtain more objective customer demands from online comment data. Besides, the improved quantitative Kano model is developed to calculate the final importance index of customer requirements.

\section{Acknowledgements}

This research was supported byKey Scientific and Technological Project of Henan Province (20210221044, 182102210442);2019 Higher Institution Youth Scholar Supporting Program of Henan Province ( 2019GGJS179;2020 Key Scientific Research Projects of Henan Higher Institutions (20A630035).

\section{Reference}

[1] X.J. Ma,"Research on customer demand analysis method of complex mechanical and electrical products for rapid customization," Southwest Jiaotong University, 2018.

[2] X.J. Li, "Product Design of Smart Home Gateway Based on User Demand," Science andTechnology Innovation, vol. 10, pp. 88-89, 2019.

[3] Y. Zhou,"Research on humanized design of products under the background of artificial intelligence," Industrial Design, no. 02, pp. 47-48, 2021.

[4] X.Z. Liu, X. Han, R. Li, H.Y. Wu, Y.S. Qian,"Method for determining the importance of subway demand for multi-agent customers," Mechanical Design and Research, vol. 35, no. 03, pp. 145-149, 2019.

[5] S.Gao,"A method for determining and ranking the importance of bus passenger demand," Kunming university of science and technology, 2014.

[6] F.L. Zhang, C.X.Jia, "Research on product innovation method based on user demand classification and importance evaluation,” Packaging Engineering, vol. 38, no. 16, pp. 87-92, 2017.

[7] M.C. Liu, H.F. Zhan, X. Shi, Y.X. Wang, "Analysis method of importance of user requirements for product design,” Machinery Manufacturing, vol. 58, no. 12, pp. 1-9, 2020.

[8] C.Y. Zhu, M.S. Yang, X.Q. Gao, X.C. Yang, "Revising the importance of customer demand based on DEMATEL and entropy,” Industrial Engineering and Management, vol. 17, no. 03, pp. 97-101, 2012.

[9] L. Yang, T,X. Wang, R.M. Zhang, J.F. Lei, Y.P. Ma,"Design evaluation and application of intelligent rice cooker based on fuzzy analytic hierarchy process," Mechanical design, vol. 36, no. 04, pp. 129-133, 2019.

[10] X.L.Geng, H.Q.Qiu,"The method of determining the importance of customerdemand considering customer satisfaction," Computer Integrated Manufacturing System, vol. 25, no. 08, pp. 2025-2035, 2019.

[11] P.Ji, J. Jin, T. Wang, Y.Z. Chen, “Quantification and integration of Kano's model into QFD for optimizing product design,” International Journal of Production Research, vol. 52, no. 21, pp. 6335-6348, 2014.

[12] Z.F. Li, J.Cai, S.X. Zhang, “Analysis of the change of user demand in different income groups under

ISSN: 0010-8189

(C) CONVERTER 2021 
the background of housing industrialization-based on Kano model," Journal of Engineering Management, vol. 33, no. 01, pp. 135-140, 2019.

[13] H.K. Geng. "Research on ranking the importance of house of quality demand based on niche and Kano model," Hangzhou dianzi university, 2020.

[14] J.Hou, M.M. Chen, "Research on B2C Website Service Quality Improvement Based on Improved QFD," Technology and Innovation Management, vol. 39, no. 03, pp. 323-331+358, 2018.

[15] Z. Wei, H.B. Shi, T. Cao, N.C. Wang, G. Zhao, D.D. Shi, "Research progress of smart wearable devices at home and abroad," Chinese Medical Equipment, vol. 17, no. 10, pp. 18-21, 2020.

ISSN: 0010-8189 